\title{
The Treatment of Orofacial Pain by Using Transcranial Direct Current Stimulation
}

\author{
J. FRICOVÁ ${ }^{1,2}$, K. ENGLEROVÁ ${ }^{1}$, J. NEDVÍDEK $^{1}$, R. ROKYTA ${ }^{1}$ \\ ${ }^{1}$ Department of Physiology, Third Faculty of Medicine, Charles University, Prague, Czech \\ Republic, ${ }^{2}$ Department of Anesthesiology, Resuscitation and Intensive Medicine, First Faculty of \\ Medicine and General University Hospital, Charles University, Pain Management Center, Prague, \\ Czech Republic
}

Received March 21, 2019

Accepted October 24, 2019

\section{Summary}

Neurostimulation methods are used in the treatment of chronic pain, although mainly for pharmacology resistant pain. Transcranial Direct Current Stimulation (tDCS) is a non-invasive neurostimulation method using low direct current (0.029-0.08 mA $\left./ \mathrm{cm}^{2}\right)$ applied to a cathode and anode, which directly stimulates the cranial surface. The applied current causes the most significant changes directly under the electrodes: the cathode reduces the excitability of cortical neurons, whereas the anode increases excitability. The effect of stimulation usually lasts a few hours up to a few days. We observed 19 patients with chronic orofacial pain. Inclusion criteria for the study were the following: orofacial pain, stable analgesic medication for at least one week before the beginning of stimulation and during its course, and age 18-75 years old. Patients with severe organic brain damage or seizure disease (epilepsy) were not included. The most common diagnosis was secondary trigeminal neuralgia after dental surgery. We measured thermal and tactile stimulation on the face before and after tDCS, then at 14 days. The total follow-up period lasted six months. We evaluated pain on a numerical scale (0-10) at each follow-up. We used sets of inventories focused on the examination of pain (a short form of McGill inventory), depression, anxiety, and pain interference with daily activities. tDCS is a non-invasive stimulation technique that is affordable and can be easily administered, especially when compared to other neurostimulation techniques. Only 15 patients out of the total number of 19 responded to the questionnaires.

\section{Key words}

Transcranial Direct Current Stimulation • Orofacial pain • Pain scale • Psychological evaluation

\section{Corresponding author}

R. Rokyta, Department of Physiology, Third Faculty of Medicine, Charles University, Prague, Czech Republic. E-mail: richard.rokyta@If3.cuni.cz

\section{Introduction}

In the treatment of pain, neurostimulation methods are most beneficial to patients suffering from intractable chronic pain, in particular chronic pain that is unresponsive to other long-term treatments (Simpson 2003). The tDCS (transcranial stimulation by direct current) neurostimulation method uses a cathode and anode, which are placed on the head, that deliver low direct current $\left(0.029-0.08 \mathrm{~mA} / \mathrm{cm}^{2}\right)$ to the cranial surface, i.e. direct current applied transcranially. tDCS is a noninvasive stimulation technique that is affordable and easily controlled compared to other neuromodulation techniques (Medeiros 2012). Anode stimulation increases cortical excitement, while cathode stimulation decreases cortical excitement. tDCS is a promising method for chronic pain management (O'Connell 2015); it may also be useful in patients with neuropsychiatric and neurologic diseases (Medeiros 2012, Kuo et al. 2013).

In tDCS, only a small amount of current penetrates through to the underlying cortex. The applied current has the greatest effect directly under the electrodes. The effect of stimulation can be changed by changing the electrode surface area and current density. The effect of stimulation usually lasts a few hours.

Prevalence of orofacial pain varies significantly 
and depending on the study, it affects $10-50 \%$ of the adult population (Johannes et al. 2010). Orofacial pain is often associated with a history of gingiva and tooth pain (MacFarlane 2014). The most common cause of acute orofacial pain is a toothache. Chronic facial pain can be unilateral or bilateral with either a permanent or episodic course. Another common cause of orofacial pain is a toothache, which can appear after dental treatment or dental surgery. According to the international classification, it is atypical odontalgia; patients are very often registered under the wrong diagnosis, i.e. under atypical orofacial pain, which is usually pharmacotherapy resistant. Chronic pain that emerges after dental treatment, or dental surgery is often intractable. Recent studies (Rokyta and Fricova 2014, Fricova et al. 2013) have suggest a possible mechanism for atypical odontalgia is a pathophysiology involving the peripheral and central nervous systems. The origin of orofacial pain can be the temporomandibular joint (TMJ), which involves pain during yawning, chewing, and singing, additionally, the temporomandibular joint is usually sensitive to touch. The pain is dull, permanent, and unilateral. The pain is of medium intensity and is more intensive when the temporomandibular joint is moving. Movement of the TMJ can also be limited by trismus. TMJ pain often subsides after massage and rehabilitation, although, in some cases, surgical treatment is necessary.

\section{Methods}

Our study is in accordance with the Declaration of Helsinki (2000) of the World Medical Association. It was approved by Ethic Committee of Third Faculty of Medicine of Charles University.

We used a protocol similar to that used in our previous study (Fricova et al. 2016); however, we included more patients in this study; the total number was 19. From these study there no difference between males and females therefore we used the both sexes together. This time we focused not only on an objective evaluation but also on an evaluation of the McGill inventory of pain descriptors and the Beck depression and anxiety inventory.

Before entering the study, patients were informed, in detail, about its structure, clinical course, and the possible effect of treatment. As part of the informed consent, it was necessary to be familiar with the side effects and potential complications of the therapy. Participation in the study was voluntary and without any financial reward. Study inclusion criteria were as follows: (a) intractable orofacial pain diagnosed by a specialist, (b) stable analgesic medication for at least one week before the study and throughout its course, (c) 18-75 years of age, (d) absence of severe organic brain damage or other serious diseases that could be impacted by tDCS (e.g. epilepsy), and (e) an available MRI (not older than two years). Based on these criteria, 19 patients with chronic orofacial pain, i.e. secondary trigeminal neuralgia after oral surgery, were enrolled and underwent both anode and cathode stimulation. Before the first session we measured pain intensity on a numeric pain rating scale, and tactile and thermal stimulations were used to assess somatosensory status. tDCS was applied for five consecutive days. At the end of tDCS treatment, somatosensory status was assessed again. Before starting tDCS stimulation, patients were assessed using a variety of inventories (e.g. McGill University and Beck Inventory). We felt that it was very important to know the patient's subjective pain description and the subjective feelings of patients with regard to the impact of chronic pain on their daily lives. The HDC stim device (Newronica) was used to administer the tDCS. The patients were divided into two groups. Group 1 underwent actual tDCS, while Group 2 underwent sham stimulation. After each session, the type of stimulation was switched, which means that ultimately all patients underwent both real and sham stimulation. The length, amplitude, the interval between the two stimulations were the same. In each session, we used the following parameters: $1 \mathrm{~mA}, 20 \mathrm{~min}$ of stimulation. The cathode and anode were placed on the affected temporal side of the skull and represented the projection of the dorsolateral prefrontal (DLPF) cortex.

Pain was assessed using a visual analog scale (VAS) (Khedr et al. 2005), which is a subjective $0-10$ point scale ( 0 representing the absence of pain and 10 being maximum pain) and by quantitative sensory testing (QST) using objective assessment instruments that set the threshold for thermal and tactile (touch) sensation in the affected facial area (Fricova et al. 2013). Thermal sensation was assessed using a specially modified device that created increasing irritation with a flow of warm air (the temperature of which ranged between $44-55^{\circ} \mathrm{C}$ ) and mechanical sensitivity, specifically, the tactile threshold was determined using von Frey hairs (Touch-test sensory evaluators).

Additional measurements assessing the influence of depression and anxiety symptoms were made using the 
Beck Depression Inventory (BDI) and by the Beck Anxiety Inventory (BAI).

To assess trends, linear regression was used; the paired $t$-test was used to evaluate changes (in the absence of a normal distribution, the Wilcox test was used).

\section{Results}

A VAS (Visual analog pain scale - axis $\mathrm{x}$ ), was used before stimulation, after series of five stimulations, and 14 days after last stimulations. Based on the VAS, $62.5 \%$ of patients reported that tDCS reduced pain perception by $53.7 \pm 31.5 \%, 12.5 \%$ reported that the pain was unchanged, and $25 \%$ reported that the pain had worsened by $53.3 \pm 3.33 \%$. The difference between active (solid line) and sham (dashed line) stimulations is seen in Figure 1. Pain relief (axis y) can be seen to last 14 days after stimulation, while in the sham group of patients, the pain returned to its original intensity by day 14 (Fig. 1).

For each patient, the trend in self-reported pain for each type of pain was marked and the graph includes total resultant trends (negative $=$ the pain decreases (green) and positive, pain increases (red)). Patients (5, 7, 9, and 14) had a statistically significant decrease in pain. Patient No. 13 had a statistically significant increase in pain.
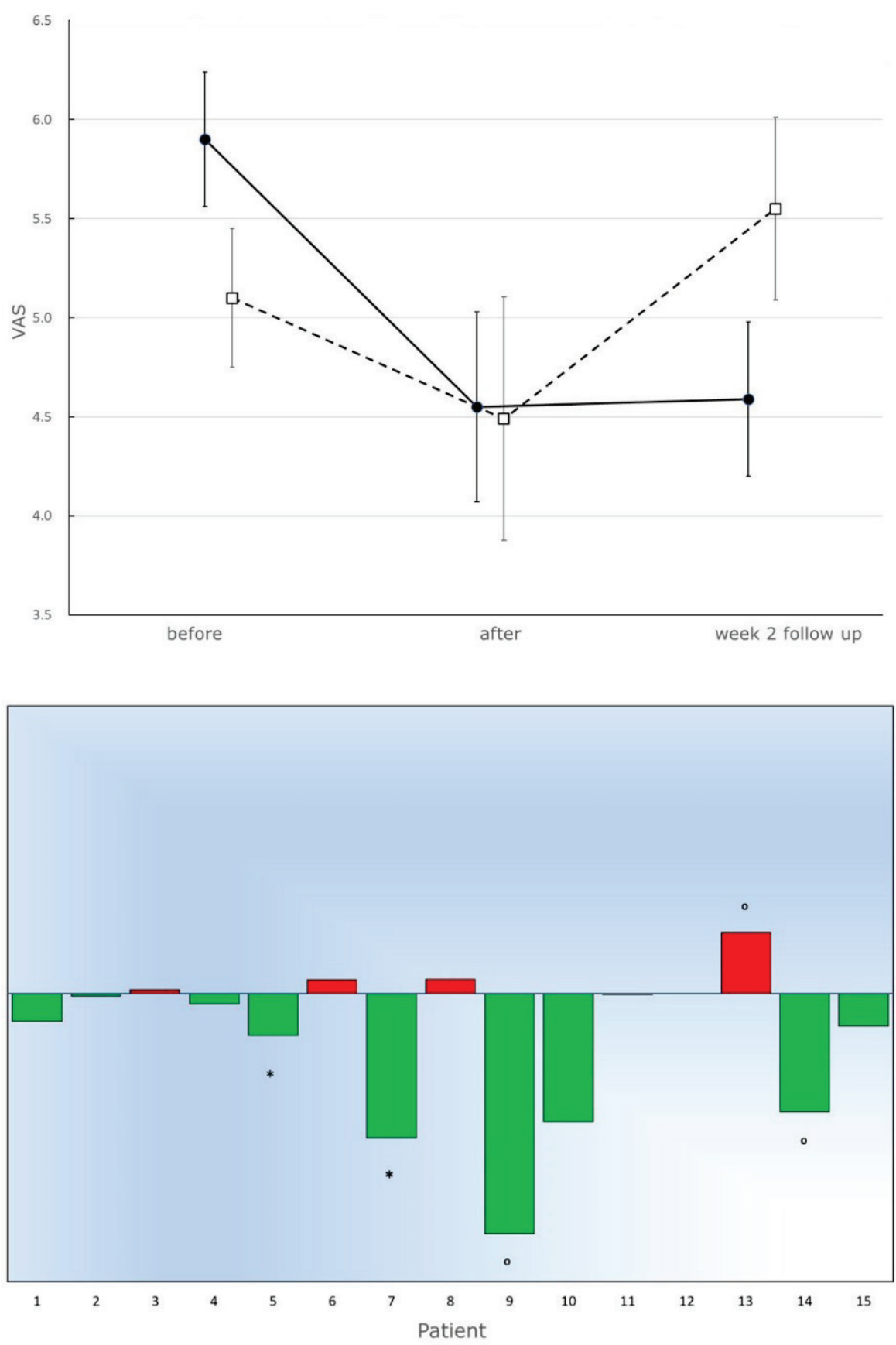

Fig. 1. VAS pain scale, before stimulation, after 5 sessions of stimulations and after 14 days. Solid line active stimulation, dashed line sham stimulation.

Fig. 2. McGill University inventory, evaluation of patients - according to trends. ${ }^{\circ} \mathrm{P}<0.05,{ }^{*} \mathrm{P}<0.001$. 

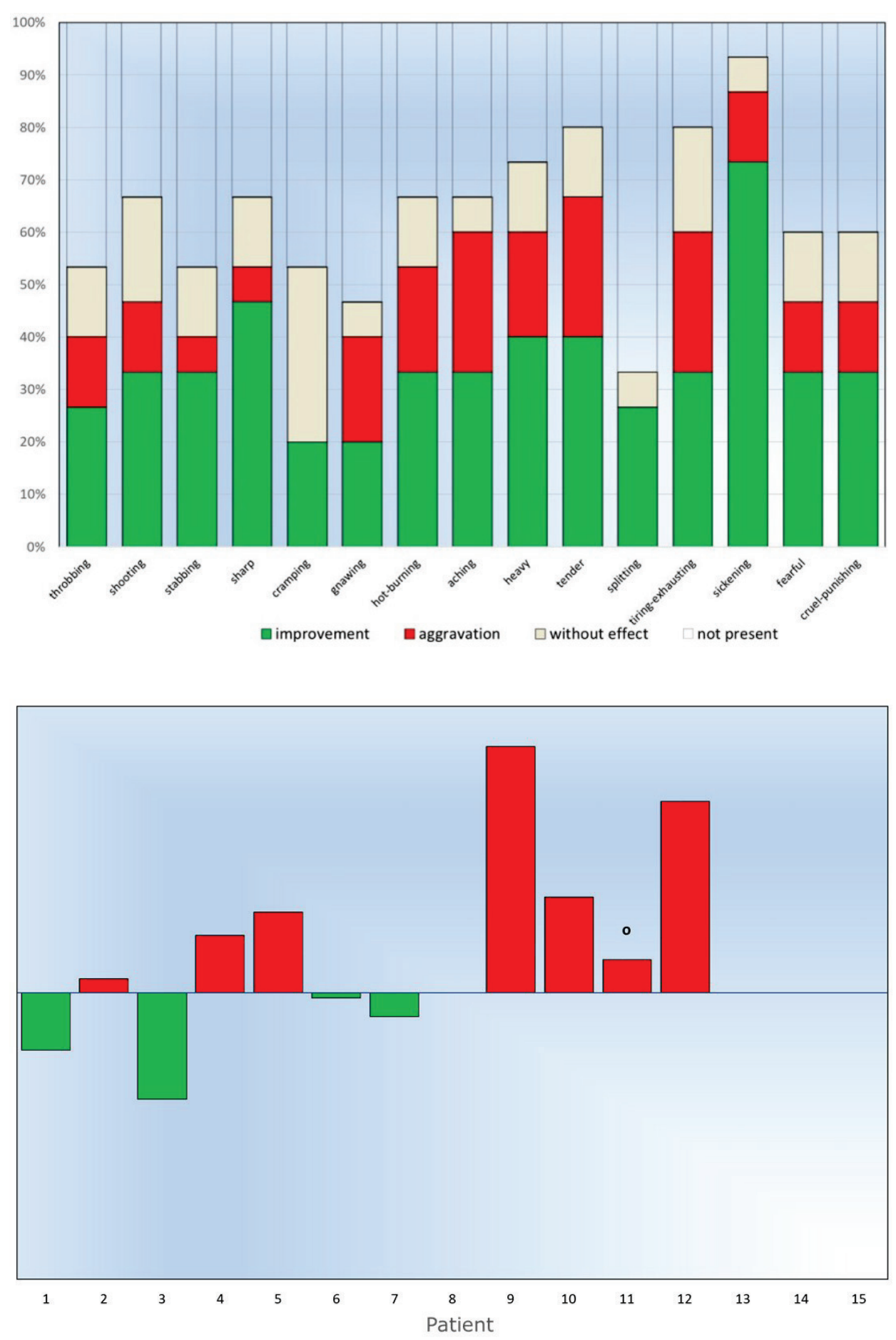

Fig. 3. McGill University questionnaire, pain descriptors evaluation of patients - according to trends.
Fig. 4. Beck depression inventtory - evaluation of patients according to trends. ${ }^{\circ} \mathrm{P}<0.05$.
Based on pain descriptors, we counted in what percentage the intensity of a certain type of pain decreased, in what percentage it was unchanged, and in what percentage the pain worsened. Only positive/negative/no effect is taken into account regardless of the size of the change. Without any effect means that a patient felt a certain type of pain with the same intensity. It is not is the percentage of patients who did not report a certain type of pain at all. e.g. pain like crack is not present in $66.7 \%$ patients at all, but in annoying pain, it is only in $6.7 \%$.

According to the Beck depression inventory of self-reported depression grade, worsening of depression symptoms appeared in 7 patients and in 4 patients depression symptoms improved.

According to the Beck anxiety inventory of selfreferred anxiety, improvement of anxiety symptoms appeared in 9 patients, with significant improvement in 4, while 3 patients had their anxiety symptoms slightly deteriorate. 


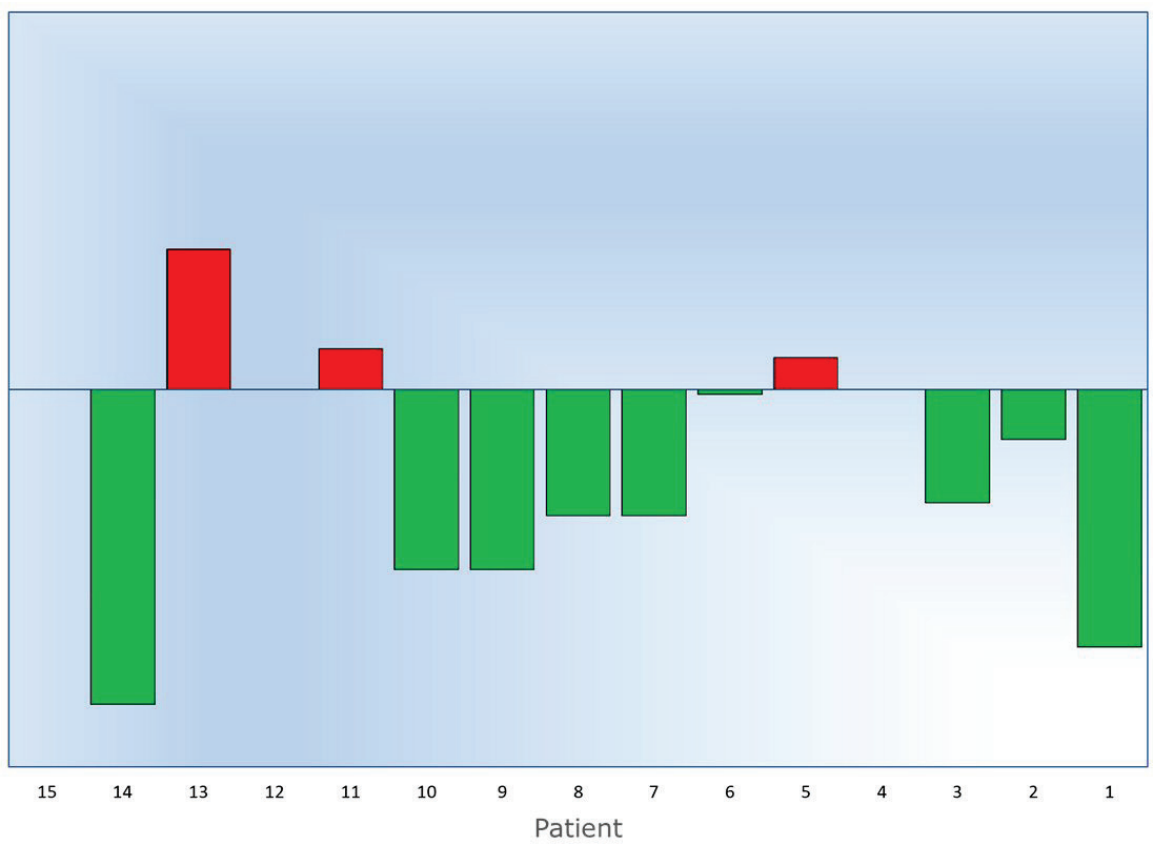

Fig. 5. Beck anxiety inventory evaluation of patients - according to trends.

\section{Discussion}

During our observation of the effect of tDCS on chronic orofacial pain, we found that orofacial pain usually decreases after the application of tDCS and the decrease lasts 14 days, sometimes even longer. At the same time, other psychological components of pain also seem to change. These components were measured using the Beck self-assessment scale (Beck inventory depression and Beck inventory anxiety). It is interesting that symptoms of depression slightly worsen after tDCS stimulation whereas anxiety symptoms significantly improve. tDCS has a significant effect on self-reported pain in patients, who mostly reported improvement in pain even if it was not accompanied by significant objective changes in pain (i.e. thermal and tactile sensations).

The course of pain episodes and the localization in the region of the face supplied by trigeminal nerve are typical for trigeminal neuralgia. The pain lasts for several seconds or minutes and is usually described as a throbbing pain with a strong intensity, unilateral, and localized to the orbital or mandibular region face. Trigeminal neuralgia can be caused by nerve compression caused by a vessel (neurovascular conflict), which can be treated by neurosurgery. Post-herpetic neuralgia can also cause facial pain. Pain usually lasts six weeks but can last longer than four months, and in up to $50 \%$ of patients, it occurs after an acute case of shingles caused by reactivation of the herpes zoster virus. It affects mostly people older than 50 . The pain is permanent; its character is usually described as burning or throbbing with a strong intensity.

A precise differential diagnosis of orofacial pain requires imaging methods. Additionally, a detailed dental and ENT examination is crucial for a proper assessment. To date, few clinical studies on the diagnosis of orofacial pain have been performed (Zakrzewska 2013). From a clinical point of view, the most transparent aid is to divide pain into permanent or episodic and according to localization into unilateral or bilateral. It is also possible to use conventional classifications and describe organic pain as neuropathic or vascular. From a diagnostic point of view, we should always remember that orofacial pain can be a primary or secondary symptom of carcinoma or metastasis. However, most patients with orofacial pain are placed under the G50.1 diagnosis, i.e. atypical orofacial pain with no unambiguous explanation for the organic origin of the pain. In this case, the terminology is slightly misleading, and a diagnosis is often based on the scarcity of evidence. Secondary trigeminal neuralgia, having a significant affective and emotional component to the pain is usually hidden under this diagnosis.

\section{Conclusions}

tDCS is a promising method of treatment for chronic orofacial pain. In future projects, we would like to study the effect of tDCS on glial cells and its effect on neuroplasticity and neuroprotection. We would also like to evaluate the effect of tDCS on phantom pain, back pain, and eventually fibromyalgia. From our results, we 
can conclude that the application of tDCS improves the perception of some types of pain, especially chronic orofacial pain. When we increase our sample size, we expect confirmation not only of our positive results, but also findings that will explain the pathophysiology of orofacial pain.

\section{Conflict of Interest}

There is no conflict of interest.

\section{Acknowledgements}

The paper was supported by Charles University grant "Progress Q 35" and the Agency of Health Research of the Ministry of Health grant "AZV 15-31538 A".

\section{References}

FRICOVÁ J, ENGLEROVÁ, ROKYTA R: Noninvasive transcranial direct current stimulation (tDCS) for the treatment of orofacial pain. Neuroendocrinol Lett 37: 101-105, 2016.

FRICOVÁ J, KLÍROVÁ M, MASOPUST V, NOVÁK T, ROKYTA R: Repetitive transcranial magnetic stimulation in the treatment of chronic orofacial pain. Physiol Res 62 (Suppl 1): S125-S134, 2013.

JOHANNES CB, LE TK, ZHOU X, JOHNSTON JA, DWORKIN RH: The prevalence of chronic pain in United States adults: results of an Internet-based survey. J Pain 11: 1230-1239, 2010.

KHEDR EM, KOTB H, KAMEL NF, AHMED MA, SADEK R, ROTHWELL JC: Longlasting antalgic effects of daily sessions of repetitive transcranial magnetic stimulation in central and peripheral neuropathic pain. $J$ Neurol Neurosurg Psychiatry 76: 833-838, 2005.

KUO HI, BIKSON M, DATTA A, MINHAS P, PAULUS W, KUO MF: Comparing cortical plasticity induced by conventional and high-definition $4 \times 1$ ring tDCS: a neurophysiological study. Brain Stimul 6: 644-648, 2013.

MACFARLANE TV, BEASLEY M, MACFARLANE GJ: Self-reported facial pain in UK biobank study: prevalence and associated factors. J Oral Maxillofac Res 5: e2, 2014.

O’CONNELL NE, WAND BM: Transcranial direct current brain stimulation for chronic pain. BMJ 350: h1774, 2015.

ROKYTA R, FRICOVÁ J: Neurostimulation methods in the treatment of chronic pain. Physiol Res 61 (Suppl 2): S23-S31, 2012.

SIMPSON B: Selections of patients and assessment. In: Electrical Stimulation and the Relief of Pain. Elsevier, Amsterdam, 2003, pp 161-182.

ZAKRZEWSKA JM: Differential diagnosis of facial pain and guidelines for management. Br J Anaesth 111: 95-104, 2013. 